\title{
Operational Evaluation of Certain Infinite Bessel Function Integrals
}

\author{
By Stanley E. Babb, Jr. and James W. Cafky
}

Abstract. Some infinite integrals, primarily over trigonometric functions, are operationally evaluated by two extensions of the Weber-Schafheitlin approach.

Introduction. In a recent investigation [1] it was necessary to numerically evaluate the integral

$$
\int_{0}^{\infty} \frac{G(u) \cos u t d u}{1-\lambda G(u)}
$$

where

$$
G(u)=\frac{\sin u / u-\cos u}{u^{2}}
$$

for a large number of values of $t$. To reduce the long range behavior and improve convergence markedly one can subtract $\lambda^{n}$ times the first several integrals

$$
I_{n}(t)=\int_{0}^{\infty} G^{n}(u) \cos u t d u
$$

but this technique requires the analytic evaluation of the integrals (3) for several low values of $n$. While the case $n=1$ is a standard Weber-Schafheitlin form, higher values of $n$ seem not to have been worked out, although it is known [2] that $I_{n}(t)=0, t>n$. Two methods have been devised for evaluating this and similar integrals, each with its own range of applicability.

Method. For the $I_{n}$ the method consists of expanding the $G^{n}$ and using multiple angle trigonometric identities to reduce the powers of the functions to unity. These resulting functions are converted to spherical Bessel functions, which replaces the original integrand with a series of products of two such functions. Each individual pair is treated as if the integral over it were convergent alone, and the standard Weber-Schafheitlin formulas [3] are used to convert the integrals to hypergeometric functions, which for this case all truncate to finite series. Terms are then gathered for the final answer.

To illustrate the technique the evaluation of $I_{2}$ and $I_{3}$ will be presented in skeletal form. Thus, after expansion and recombination

Received June 26, 1978.

AMS (MOS) subject classifications (1970). Primary 42A68, 44A20.

Key words and phrases. Fourier transforms, Bessel functions. 


$$
\begin{aligned}
I_{2}=\frac{\pi}{2} \sqrt{t / 2} \int_{0}^{\infty}\{ & \frac{J_{0}(a u) J_{-1 / 2}(u t) \sqrt{u}}{\sqrt{\pi u^{4}}}+\frac{J_{0}(a u) J_{-1 / 2}(u t) \sqrt{u}}{\sqrt{\pi u^{6}}} \\
& \left.+\frac{J_{1 / 2}(2 u) J_{-1 / 2}(u t)}{u^{3}}-\frac{J_{1 / 2}(2 u) J_{-1 / 2}(u t)}{2 u^{4}}-\frac{J_{-1 / 2}(2 u) J_{-1 / 2}(u t)}{u^{5}}\right\} d u,
\end{aligned}
$$

where a $J_{0}(a u)$ has been inserted in the first two terms to get the proper form, and after integrating, then $a$ is set to 0 . This procedure is not essential to the method but does avoid the introduction of subsidiary procedures. Similarly for $I_{3}$,

$I_{3}=\frac{\pi}{8} \sqrt{t} \int_{0}^{\infty}\left\{\frac{3 J_{1 / 2}(u) J_{-1 / 2}(u t)}{u^{8}}-\frac{\sqrt{3} J_{1 / 2}(3 u) J_{-1 / 2}(u t)}{u^{8}}-\frac{3 J_{-1 / 2}(u) J_{-1 / 2}(u t)}{u^{7}}\right.$

$$
\begin{array}{r}
+\frac{3 \sqrt{3} J_{-1 / 2}(3 u) J_{-1 / 2}(u t)}{u^{7}}+\frac{3 J_{1 / 2}(u) J_{-1 / 2}(u t)}{u^{6}}+\frac{3 \sqrt{3} J_{1 / 2}(3 u) J_{1 / 2}(u t)}{u^{6}} \\
\left.-\frac{3 J_{-1 / 2}(u) J_{-1 / 2}(u t)}{u^{5}}-\frac{\sqrt{3} J_{-1 / 2}(3 u) J_{-1 / 2}(u t)}{u^{5}}\right\} d u,
\end{array}
$$

and now each individual term is handled by using the Weber-Schafheitlin formulas.

No completely general proof of the method, applicable to all cases herein considered, has yet been derived, although the general case for a linear combination of two Bessel functions multiplied by a single Bessel function has been worked out.

The proofs must, therefore, be performed for each individual case or assumed. The methods involve the standard proof of the Weber-Schafheitlin formulas [4], where the various terms in the expansions therein encountered which diverge at the origin cancel each other. After integration of the series, the first several terms of the hypergeometric functions also cancel and correspond exactly to the cancelling terms of the original expansions. Thus, the equivalence can be established in each case, although the details can be quite tedious.

In practice, the method is applied operationally. Generally speaking, if any of the functions indicated by this symbolic procedure do not exist, then one of the assumed cancellations does not take place; and the procedure breaks down. Either a reformulation of the terms into Bessel functions is indicated, or the integral must be done by other techniques (see below). As a check on the algebra, $I_{n}(t)$ for $t>n$ will be found to be identically zero, also $I_{n}(t)$ is continuous.

The algebra rapidly becomes quite tedious, but the results which have been generated to date are presented here in hope of their utility:

$$
\begin{aligned}
I_{1} & =-\frac{\pi}{4}\left(1-t^{2}\right), \quad 0 \leqslant t \leqslant 1 \\
& =0, \quad t \geqslant 1
\end{aligned}
$$




$$
\begin{aligned}
& I_{2}=\frac{\pi}{2}\left(\frac{2}{15}-\frac{t^{2}}{6}+\frac{t^{3}}{12}-\frac{t^{5}}{240}\right), \quad 0 \leqslant t \leqslant 2, \\
& =0, \quad t \geqslant 2 \text {, } \\
& I_{3}=\frac{-\pi}{2^{6}}\left(\frac{141}{120}-\frac{5 t^{2}}{6}+\frac{t^{4}}{4}-\frac{t^{6}}{30}+\frac{t^{8}}{2520}\right), \quad 0 \leqslant t \leqslant 1 \text {, } \\
& =\frac{-\pi}{2^{6}}\left(\frac{81}{80}+\frac{27 t}{35}-\frac{9 t^{2}}{4}+\frac{6 t^{3}}{5}-\frac{t^{4}}{8}-\frac{t^{5}}{15}+\frac{t^{6}}{60}-\frac{t^{8}}{5040}\right), \quad 1 \leqslant t \leqslant 3, \\
& =0, \quad t \geqslant 3 \text {, } \\
& I_{4}=\frac{\pi}{15}\left(\frac{167}{2079}-\frac{17 t^{2}}{378}+\frac{t^{4}}{84}-\frac{7 t^{6}}{2880}+\frac{t^{7}}{1792}+\frac{t^{8}}{32256}\right. \\
& \left.-\frac{t^{9}}{64512}+\frac{t^{11}}{14192640}\right), \quad 0 \leqslant t \leqslant 2 \\
& =\frac{\pi}{45}\left(\frac{64}{693}+\frac{16 t}{35}-\frac{44 t^{2}}{63}+\frac{t^{3}}{3}-\frac{t^{4}}{28}-\frac{t^{5}}{48}\right. \\
& \left.+\frac{7 t^{6}}{960}-\frac{t^{7}}{1792}-\frac{t^{8}}{10752}+\frac{t^{9}}{64512}-\frac{t^{11}}{14192640}\right), \quad 2 \leqslant t \leqslant 4 \\
& =0, \quad t \geqslant 4 \text {, } \\
& I_{5}=\frac{\pi}{2654208}\left(-\frac{6891623}{1617}+\frac{40949 t^{2}}{21}-\frac{14697 t^{4}}{35}+\frac{281 t^{6}}{5}\right. \\
& \left.-5 t^{8}+\frac{9}{35} t^{10}-\frac{t^{12}}{385}+\frac{t^{14}}{175175}\right), 0 \leqslant t \leqslant 1 \\
& =\frac{\pi}{86016}\left(-\frac{3444179}{24948}-\frac{3727}{6435} t+\frac{21209}{324} t^{2}-\frac{4577}{891} t^{3}\right. \\
& -\frac{373}{60} t^{4}-\frac{562}{81} t^{5}+\frac{3227}{540} t^{6}-\frac{10}{7} t^{7}+\frac{t^{8}}{108} \\
& \left.+\frac{19 t^{9}}{405}-\frac{t^{10}}{180}-\frac{t^{11}}{4455}+\frac{t^{12}}{17820}-\frac{t^{13}}{8108100}\right), \quad 1 \leqslant t \leqslant 3 \\
& =\frac{\pi}{111476736}\left(\frac{7421875}{77}-\frac{95625000}{143} t+734375 t^{2}-\frac{3275000}{11} t^{3}\right. \\
& +16875 t^{4}+26000 t^{5}-8925 t^{6}+\frac{6480}{7} t^{7}+93 t^{8} \\
& \left.-\frac{152}{5} t^{9}+\frac{9}{5} t^{10}+\frac{8}{55} t^{11}-\frac{t^{12}}{55}+\frac{t^{14}}{25025}\right) \\
& =0, \quad t \geqslant 5 \text {. }
\end{aligned}
$$


Since $G(u)=-j_{1}(u) / u$, these results are the Fourier transforms of these functions to the powers 1 through 5 .

Though developed for use with the $G(u)$ functions, this technique is by no means limited to them. Thus, for example,

$$
\begin{gathered}
\int_{0}^{\infty} \frac{\sin ^{2} u \sin ^{3} 2 u}{u^{5} \cos u t d t=\pi\left(544-48 t+\frac{3 t^{4}}{2}\right), \quad 0 \leqslant t \leqslant 2,} \\
=\frac{\pi\left(496+96 t-120 t^{2}+24 t^{3}-\frac{3 t^{4}}{2}\right)}{284}, \quad 2 \leqslant t \leqslant 4, \\
=\frac{\pi\left(752-160 t-24 t^{2}+8 t^{3}-\frac{t^{4}}{2}\right)}{284}, \quad 4 \leqslant t \leqslant 6, \\
=\frac{\pi\left(2048-1024 t+192 t^{2}-16 t^{3}+\frac{t^{4}}{2}\right)}{284}, \quad 6 \leqslant t \leqslant 8, \\
=0, \quad t \geqslant 8 .
\end{gathered}
$$

It is not necessary that the hypergeometric functions truncate, e.g. the known integral

$$
\int_{0}^{\infty}(\sin a x \sin b x) \frac{d x}{x}=\ln \left(\frac{a+b}{a-b}\right)^{2} / 4
$$

is easily done being a standard Weber-Schafheitlin form. When, however, the answers do not lend themselves readily to hypergeometric series, the method fails, e.g. no obvious way exists to contort the integrand of

$$
\begin{aligned}
& \int_{0}^{\infty}\left(\sin ^{2} a x \sin ^{2} b x\right) \frac{d x}{x^{3}} \\
& \quad=\left(a^{2}+b^{2}\right) \ln \left(b^{2}-a^{2}\right)+a b \ln \left(\frac{b+a}{b-a}\right)-b^{2} \ln b-\left(a^{2} \ln a\right) / 2
\end{aligned}
$$

(for $b>a$ ) into a form which permits the last two log terms. The attempts to use the Weber-Schafheitlin formulation results in terms which do not exist, indicating that an assumed cancellation does not occur. If the power of $x$ in the denominator were either 2 or 4 , the method yields known results.

For integrals of this last type it has proved possible to develop operational procedures for evaluation under somewhat more limited conditions. Providing the integral can be written in the form $\left[\Sigma_{i} J_{\nu_{i}}\left(b_{i} u\right)\right] J_{\mu}(u t)$, where $t>$ all $b_{i}$ a procedure exists. Thus, the integrand mentioned in the previous paragraph could be written in the form $\sin ^{2} a x \sin b x \sin c x$, where $c>a, b$, and is then evaluated by the procedure outlined below. After reduction to an answer involving only elementary functions, $c$ can be set equal to $b$ without problems. The procedure is derived from that used for the standard Weber-Schafheitlin proof in Watson [4], and is essentially obvious from that proof. 
If one defines the function

$$
F c(n, b, c, z)=\frac{\Gamma(c)}{\Gamma(b)} \sum_{k=n+1}^{\infty} \frac{\Gamma(k+2-n) \Gamma(k+b)}{\Gamma(k+c)} \frac{z^{n}}{n !}
$$

(which can be written as a generalized hypergeometric function, but this gains nothing for the present purposes), then the individual terms can be evaluated by

$$
\begin{gathered}
\int_{0}^{\infty} \frac{J_{\alpha+\rho}(a u) J_{\alpha-\rho-1}(u t) d u}{u^{\lambda}} \\
=\frac{a^{\alpha+\rho}}{2^{\lambda} t^{\alpha+\rho-\lambda+1} \Gamma(\alpha+\rho+1) \Gamma(\lambda / 2-\rho)} F c\left(\frac{\lambda}{2}-\alpha, \rho+1-\frac{\lambda}{2}, \alpha+\rho+1, \frac{a^{2}}{t^{2}}\right), \\
\alpha-\frac{\lambda}{2}=-n,
\end{gathered}
$$

where it must be strongly emphasized that this type of evaluation is only correct within the current context. The first few terms of the standard expression cancel each other, which is signalled by $\alpha-\lambda / 2=-n$; and it is the remainder which are given by the expressions above. The procedure is now to apply this expression to each term of the integrand and then evaluate the summations. The four gamma functions in front of the summations reduce to $\pm 1 / \pi$, and the summations themselves can be broken by partial fractions into sums of the form $x^{k} / k$ or $x^{k} /(2 k+1)$ and, thus, are easily done.

If $t<$ any term in the integrand sum, then the same type of procedure based upon the usual formulas for $t<a$ may not work. The terms which can enter are not necessarily easily expressible as either ${ }_{2} F_{1}$ or $F c$ functions, and may arise from several terms acting in concert. No satisfactory operational technique for this case has yet been devised. Fortunately, the integrals herein considered can be written in forms which are continuous in $a$ and $b$ (excepting singularities).

Attention has been focused upon the integrals

$$
J_{n}=\int_{0}^{\infty} u G^{n}(u) \cos u t d u,
$$

where, as in the case of the $I_{n}$, the first case is a standard form and the others have been worked out by the above procedure. The answers derived so far are

$$
\begin{aligned}
J_{1}=-1+ & \frac{t}{4} \ln \left(\frac{1+t}{1-t}\right)^{2} \\
J_{2}=1 / 4+ & \frac{t^{2}}{24}-\frac{t}{12} \ln \left(\frac{2+t}{2-t}\right)^{2}-\frac{t^{2}}{16}\left(1-\frac{t^{2}}{12}\right) \ln \left(\frac{t^{2}-4}{t}\right)^{2} \\
J_{3}=-\frac{3}{16} & \left\{\ln \left(\frac{t^{2}-1}{t^{2}-9}\right)^{2}\left(\frac{9}{280}+\frac{3 t^{2}}{20}-\frac{t^{4}}{72}\right)+\ln \left(\frac{t+1}{t-1}\right)^{2}\left(\frac{17 t}{144}+\frac{t^{3}}{16}-\frac{t^{5}}{80}+\frac{t^{7}}{5040}\right)\right. \\
& \left.+\ln \left(\frac{t+3}{t-3}\right)^{2}\left(\frac{-3 t}{16}-\frac{t^{3}}{48}+\frac{t^{5}}{240}-\frac{t^{7}}{15120}\right)+\frac{322}{735}+\frac{224 t^{2}}{2205}+\frac{2 t^{4}}{945}\right\}
\end{aligned}
$$


Though derived for use with trigonometric functions, both of the above methods can be applied to other integrands. Thus, for example,

$$
\begin{aligned}
& \int_{0}^{\infty}\left[J_{0}(a x)-5 !\left(\frac{2}{a x}\right)^{5} J_{5}(a x)\right] \frac{J_{3}(b x)}{x^{5}} d x \\
& =\frac{16 b^{3} a}{2079}\left[{ }_{2} F_{1}\left(-1 / 2, \frac{-11}{2}, 4, \frac{b^{2}}{a^{2}}\right)-\frac{693}{256}{ }_{2} F_{1}\left(-1 / 2,-1 / 2,4, \frac{b^{2}}{a^{2}}\right)\right], \quad b \leqslant a, \\
& =\frac{b^{4}}{105}\left[{ }_{2} F_{1}\left(-1 / 2, \frac{-7}{2}, 1, \frac{a^{2}}{b^{2}}\right)-{ }_{2} F_{1}\left(-1 / 2, \frac{-7}{2}, 6, \frac{a^{2}}{b^{2}}\right)\right], \quad b \geqslant a,
\end{aligned}
$$

by the first technique, and if the divisor of $J_{3}$ be changed from $x^{5}$ to $x^{4}$, then the result is

$$
-\frac{5 b a^{2}}{96}\left[1 / 2-\frac{2}{7}\left(\frac{a}{b}\right)^{2}+\frac{11}{168}\left(\frac{a}{b}\right)^{4}\right], \quad b>a,
$$

by the second technique.

Unfortunately, this last integral requires a different expression for $b<a$, and no operational procedure for deriving the answer

$$
-\frac{b^{3}}{24}\left[\frac{137}{240}-\frac{5}{4}\left(\frac{b}{2 a}\right)^{2}+2\left(\frac{b}{2 a}\right)^{4}-\frac{8}{3}\left(\frac{b}{2 a}\right)^{6}+\frac{16}{7}\left(\frac{b}{2 a}\right)^{8}-\frac{32}{35}\left(\frac{b}{2 a}\right)^{10}\right]
$$

has been devised. The above expression was derived by following the standard WeberSchafheitlin proof with the obvious modifications needed for this particular expression. This involves considerably more involved manipulations than the operational procedures outlined earlier. For more complex integrands this type of procedure becomes extremely involved.

The defects of the methods outlined herein are obvious. Lacking a general proof of applicability, either the methods must be viewed as simply operational, or the laborious proofs must be exercised for each particular case. When viewed as an operational technique, however, these methods have been very useful in evaluating some difficult integrals, and it is presented in the hope of utility to others.

As a final comment, one should note that some of these integrals are very similar to the $W$ integrals considered by Katsura et al. [5] using classical residue analysis. They consider products of three and four Bessel functions for particular powers of the multiplicative $u$. Their methods are more general than those herein, albeit they require more skill in analysis to apply; and it would be quite difficult to extend their methods to more products. It appears probable that all of their integrals can be done by the techniques used here, but each must be calculated on an individual basis.

Acknowledgment. This work was supported in part by a grant from the National Science Foundation. 
1. J. W. CAFKY, Ph. D. dissertation, University of Oklahoma, 1969.

2. W. N. BAILEY, "Some infinite integrals involving Bessel functions," Proc. London Math. Soc. (2), v. 40, 1936, pp. 37-48, Eq. 10.3. Also G. N. WATSON, A Treatise on the Theory of Bessel Functions, Cambridge Univ. Press, 1966, p. 419 (16).

3. G. N. WATSON, ibid., p. 404.

4. G. N. WATSON, ibid., p. 399 ff.

5. S. KATSURA, Phys. Rev., v. 115,1959 , p. 1417; S. KATSURA \& K. NISHIHARA, J. Chem. Phys., v. 50, 1969, p. 3579; J. E. KILPATRICK, S. KATSURA \& Y. INOUE, Math. Comp., v. 21,1967, pp. 267 and 407 . 